\title{
论 文
}

\section{南海深水多次波成像}

\section{刘伊克 ${ }^{(1)}$, 朱伟林 ${ }^{(2)}$ ，米立军 ${ }^{(2)}$, 周家雄 ${ }^{(2)}$, 胡昊 ${ }^{(1)}$}

(1) 中国科学院地质与地球物理研究所, 北京 100029 ;

(2) 中国海洋石油总公司, 北京 100010

* 联系人, E-mail: zhuwl@cnooc.com.cn

收稿日期: 2014-01-05; 接受日期: 2014-06-10; 网络版发表日期: 2015-01-21

国家重点基础研究发展计划项目(编号: 2009CB219405)、国家自然科学基金项目(批准号: 40930421, 41074091)和国家油气重大专项项目 (编号: 2011ZX05008-006)资助

\begin{abstract}
摘要南海海域水深可达 $5 \mathrm{~km}$, 是我国未来油气勘探最有前景的区域. 南海海域地 震资料含有各种类型多次波, 在地震成像前需将多次波去除. 然而与传统的一次反射 波偏移比较，多次波携带更多的地下结构信息，多次波偏移可提供更好的地下成像. 本文提出了一种修改传统逆时偏移的方法, 利用多次波进行成像. 该方法用包含一次 波和多次波的记录代替震源子波, 用预测多次波代替一次反射波作为输入数据. 在逆 时偏移处理流程中，将地表多次波记录沿时间延拓到各个深度层，通过互相关成像条 件将包含一次波和多次波的地震记录沿时间延拓到相同深度层. 将多次波偏移结果与 一次波偏移结果的差应用目标函数进行匹配, 弥补一次波偏移的不足. 对 Sigsbee2B 数 据做数值测试的结果表明与传统一次反射波偏移相比, 该方法可以得到范围更大、均 衡性更好的地下照明度. 将该方法应用到南海深水实际资料成像, 获得了南海深水地 震资料多次波成像剖面。偏移结果表明, 多次波成像可以弥补反射波照明的不足, 由 此说明多次波偏移是未来的一个重要研究方向.
\end{abstract}

\section{关键词}

中国南海

地震多次波逆时偏移

地震成像
南海深水勘探目标层复杂，海底崎岖，沟壑纵横, 陆坡陡峭, 需要对地下介质更准确地成像. 目前共中 心点道集 $(\mathrm{CMP})$ 叠加技术以及偏移都是基于地震数 据只含有反射波的假设条件, 即假设无多次反射, 因 而含多次波的地震数据在目前的成像算法中不是有 效信号, 而是干扰信号. 多次波会影响到速度分析及 速度模型的建立, 从而影响地震成像效果以及反演 解释过程中的介质属性提取, 最终影响勘探决策. 多 次波经一次以上的地下界面反射后最终在地面被接 收. 一般来说, 与一次反射波相比, 多次反射波在地
下传播的射线路径更长，覆盖的区域更广. 多次波常 可以传播到地球内部，照明一次反射波无法到达的 阴影区. 另外，多次反射波的反射角一般比一次反射 波小 (Berkhout和 Verschuur, 2003), 能提供更详细的 地球内部结构信息. 传统的成像方法只对一次波偏 移, 把多次波视为噪声, 而多次波偏移则利用多次波 为地下界面成像提供构造信息(Guitton, 2002; Muijs 等, 2007; Shan, 2003; Vasconcelos 等, 2008). 多次波 偏移, 最直观的方法就是将多次波转换为一次波, 然 后采用传统偏移方法(Verschuurd 和 Berkhout, 2005).

中文引用格式: 刘伊克, 朱伟林, 米立军, 等. 2015. 南海深水多次波成像. 中国科学: 地球科学, 45: 152-160

英文引用格式: Liu Y K, Zhu W L, Mi L J, et al. 2015. Migration of multiples from the South China Sea. Science China: Earth Sciences, 58: 482-490, doi: 10.1007/s11430-014-4952-y 
从多次波中直接提取有用同相轴的观点可以追溯到 (Clearbout, 1968)在地震干涉测量方面的研究. 其研 究表明如何通过地下震源激发产生的自相关道, 对 地下震源求和相加, 获得地球表面格林函数(例如一 个脉冲点源). 而这些地下震源的空间位置和激发时 间未知, 所以可以认为是一种被动地震方法. 再应用 运动学方法将多次波转换为由地表虚源产生的一次 波. 在 Claerbout 之后, 地震干涉方法被成功地运用 到垂直地震剖面(VSP)数据 (Jiang 等, 2007; Sheng, 2001; Schuster 等, 2004; Yu 和 Schuster, 2002). 另外 一种多次波偏移方法是通过最小平方偏移(Brown 和 Guitton, 2005; He 和 Schuster, 2003)将海底电缆数据 作一次反射波和鬼波成像 (Berkhout 和 Verschuur, 1994; Reiter 等, 1991)利用反馈模型和波场延拓反演, 将多次波转变为一次反射波实现多次波成像.

本文在前期研究基础上( $\mathrm{Liu}$ 等, 2011), 提出了通 过改进传统逆时偏移 (reverse time migration)的多次 波与反射波匹配方法. 我们不需要将多次波变换为 一次反射波, 用包含一次反射波和表层多次波的记 录代替数字脉冲源, 用多次波代替一次反射波, 将多 次波偏移到地下正确的位置. 在逆时偏移处理流程 中, 将地表多次波记录沿时间延拓到各个深度层, 将 包含一次波和多次波的地震记录沿时间延拓到相同 深度层. 成像条件是在各个深度层上自相关两个波 场. 多次波偏移分为两步: 第一步, 利用一些预测多 次波的方法得到多次反射波, 如 Radon 变换、预测反 禇积和表层相关多次波消除技术(SRME) (Verchuurd 等, 1992); 第二步, 利用逆时偏移进行盐下深度成像. 逆时偏移是一种基于双程波波动方程的方法, 可以 处理横向速度变化剧烈的悬垂构造.

将多次波偏移方法应用到地震实际资料是一个 非常大的挑战. 对于实际地震资料, 很难获得一个准 确的速度模型. 而对实际资料预测多次波, 需要能对 多次波的到时, 相位和振幅精确预测. 我们将多次波 偏移方法应用到南海深水实际资料, 获得了南海深水 的反射波、多次波、多次波与反射波匹配的偏移结果.

\section{1 地震多次波预测}

多次波偏移的第一步需要多次波预测. 在南海 深水资料应用了反馈迭代方法, 反馈迭代方法是一 种完全数据驱动的方法, 在迭前直接反演预测多次
波. 其基本依据是原始数据中包含了一次波和所有 与反射界面有关的多次波信息. 把这些波场作为新 的源, 并利用记录数据中所得到关于地下介质的信 息, 则可以模拟出多次波. 这个模拟过程在数学上实 现就是二维褶积或称之为 WRW 模型. 由于可供利用 的信息不完整, 所以预测多次波和实际记录中的多 次波在到时、振幅和相位上存在一些差异, 这种差异 可以通过自适应匹配滤波来消除. 地震波在地层中 的传播被看成是一个滤波过程, 如果没有自由表面 的存在, 这个滤波器的响应只有一次反射和内部层 间多次反射, 而自由表面的存在, 使得这个滤波器变 成一个带反馈的滤波器, 这样引入了自由表面多次 波, 从而可以导出关于自由表面多次波预测的迭代 公式.

$$
\begin{gathered}
M\left(z_{0}, z_{0}\right)=\sum_{n=1}^{\infty}(-1)^{n-1}\left[P^{(0)}\left(z_{0}, z_{0}\right) A\left(z_{0}, z_{0}\right)\right]^{n} P^{(0)}\left(z_{0}, z_{0}\right), \\
A\left(z_{0}, z_{0}\right)=\sum_{n=1}^{\infty}\left[S\left(z_{0}\right)\right]^{n-1}\left[R^{\wedge}\left(z_{0}, z_{0}\right) D\left(z_{0}\right)\right]^{-1},
\end{gathered}
$$

式中, $P^{(0)}\left(z_{0}, z_{0}\right)$ 表示无表面多次波数据, $M\left(z_{0}, z_{0}\right)$ 是预 测多次波数据, $A\left(z_{0}, z_{0}\right)$ 是海水面反射系数, $S\left(z_{0}\right)$ 是滤 波因子, $D\left(z_{0}\right)$ 是地震记录. (1)式表明波的传播过程在 时间空间域可以用二维褶积来表示, 在频率波数域 可以用乘积来表示. 褶积可以用矩阵来表示, 二维褶 积的矩阵表示无论在数学上的运算还是在计算上均 比较复杂. 一维褶积的矩阵表示在数学上, 在计算上 均比较简单. 此外, 采用一维褶积的矩阵表示, 波的 反向传播过程表达也比较方便. 本文应用 WRW 理论 预测出了南海多次波的地震数据.

\section{2 地震多次波偏移}

地表爆炸源激发产生地震波向地球内部传播, 介质对震源的地震响应在频率域可表示为

$$
D\left(z_{0}, z_{0}\right)=\tilde{X} S\left(z_{0}, z_{0}\right),
$$

式中, $D\left(z_{0}, z_{0}\right)$ 是接收到的地震记录, 通常包含一次反 射波和多次波; $\widetilde{X}$ 是介质响应矩阵, $S\left(z_{0}, z_{0}\right)$ 是激发子 波. 通常做法是先消除 $D\left(z_{0}, z_{0}\right)$ 中的多次波, 然后利 用一些偏移方法对一次反射波偏移.

可以将 $D\left(z_{0}, z_{0}\right)$ 当作一个虚震源, 地震波向下传 播产生一阶多次波或者更高阶的多次波 $M\left(z_{0}, z_{0}\right)$. 与 (1)式对比，可得到多次波 


$$
M\left(z_{0}, z_{0}\right)=-X D\left(z_{0}, z_{0}\right),
$$

式中, $\boldsymbol{X}$ 是地下反射界面的响应矩阵, 包括了所有一 次反射波和地下多次波. 在(4)式中, 将(3)式中的震 源和数据分别替换成 $D\left(z_{0}, z_{0}\right)$ 和 $M\left(z_{0}, z_{0}\right)$. (4)式中, 可 把波场数据视为虚震源, 它激发之后会照明地下界 面并由此生成多次波. 将包含多次波和一次波的地 震数据当作地表源, 地震波传播产生表层相关多次 波. 由反射波(一次波)产生一阶多次波, 由一阶多次 波又产生高阶多次波.

在该方法中，偏移成像的是多次波最后一个上 行反射的扰动. 如图 1 所示, $\mathrm{P}$ 点反射波作为新的震 源, 正向传播到达 $\mathrm{X} 2$, 从 $\mathrm{Mg}$ 反向传播的多次波逆向 传播到达 X4. 如果反向传播的地震波速度快于正向 传播的地震波, 正反向传播的地震波在 X3 相遇成像. 同理, $\mathrm{Mg}$ 检波器收到的一阶多次波正向传播与 $\mathrm{Mr}$ 位 置的二阶多次波反向传播在 X5 相遇成像. 每个多次 波偏移都可以通过与其低一阶的或高一阶的多次波 进行互相关成像, 或者与一次波通过互相关成像( Liu 等, 2011).

\section{3 多次波逆时偏移的成像条件}

通过对源和接收点处波场延拓得到地下界面的 方法有很多, 比如逆时偏移方法(RTM)(Baysal 等, 1983; Chang 和 Mcmechan, 1987). RTM 是全波方程, 可以准确地成像悬垂反射层和陡倾斜层. RTM 互相 关成像条件就是互相关在每个深度层的两个波场, 一个来自源, 一个来自接收点. 为了利用多次波提供 的信息, 反向延拓得到的多次波要与包含一次波、多 次波的记录经正向延拓得到的波场进行互相关.

多次波成像条件可以解释为源场与接收场之间 的零延迟互相关:

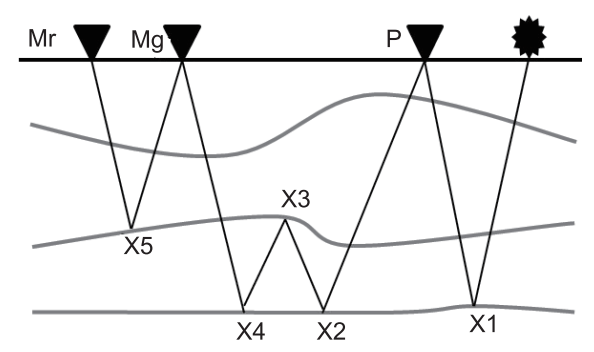

图 1 表面相关多次波偏移成像示意图

$$
\begin{aligned}
& \operatorname{Image}(x, y, z)=\sum_{t=0}^{t_{\max }}\left\{P_{F}(x, y, z, t)+M_{F}(x, y, z, t)\right\} \\
& \times M_{B}(x, y, z, t), \\
& M(x, y, z, t)=M^{1}(x, y, z, t)+M^{2}(x, y, z, t) \\
& +M^{2}(x, y, z, t)+M^{3}(x, y, z, t)+\ldots, \\
& \operatorname{Image}(x, y, z)=\sum_{t=0}^{t_{\max }}\left[\begin{array}{l}
P_{F}(x, y, z, t) \times M_{B}^{1}(x, y, z, t)+ \\
M_{F}^{1}(x, y, z, t) \times M_{B}^{2}(x, y, z, t)+ \\
M_{F}^{2}(x, y, z, t) \times M_{B}^{3}(x, y, z, t)+ \\
\cdots
\end{array}\right] \\
& +\sum_{t=0}^{t_{\max }}\left[\begin{array}{l}
P_{F}(x, y, z, t) \times M_{B}^{2}(x, y, z, t)+ \\
M_{F}^{1}(x, y, z, t) \times M_{B}^{3}(x, y, z, t)+ \\
M_{F}^{2}(x, y, z, t) \times M_{B}^{4}(x, y, z, t)+ \\
\cdots
\end{array}\right] \\
& +\sum_{t=0}^{t_{\max }}\left[\begin{array}{l}
M_{F}^{1}(x, y, z, t) \times M_{B}^{1}(x, y, z, t)+ \\
M_{F}^{2}(x, y, z, t) \times M_{B}^{1}(x, y, z, t)+ \\
M_{F}^{2}(x, y, z, t) \times M_{B}^{2}(x, y, z, t) \\
M_{F}^{3}(x, y, z, t) \times M_{B}^{1}(x, y, z, t)+ \\
M_{F}^{3}(x, y, z, t) \times M_{B}^{2}(x, y, z, t)+ \\
3, t) \times M_{B}^{3}(x, y, z, t)+\ldots
\end{array}\right] .
\end{aligned}
$$

(5)式表示成像条件, (6)式表示多次波成分, 将(6)式 代入(5)式展开为(7)式. (7)式中, Image $(x, y, z)$ 是 $(x, y, z)$ 处的像, $t_{\text {max }}$ 是全记录时间. 将包含一次反射波的数 据 $P_{F}(x, y, z, t)$ 和多次波数据 $M_{F}(x, y, z, t)$ 视为源波场 做正向延拓, 多次波场 $M_{B}(x, y, z, t)$ 反向延拓. 其中, $M(x, y, z, t)$ 表示多次波数据, $M^{1}(x, y, z, t), M^{2}(x, y, z, t)$ 和 $M^{3}(x, y, z, t)$ 分别是一阶、二阶和三阶多次波.

(7)式中, 不同乘积求和项形成真成像矩阵, 假 成像矩阵和不成像矩阵. 一般情况下, $n$ 阶多次波表 示在经过地表向下反射 $n$ 次, 在地表反射了 $2 n-1$ 次 的反射波在地表再经一次反射成为下行的虚震源能 量, 这一能量向前传播, 与反向延拓的地表反射 $2 n+1$ 次反射波后的结果进行互相关, 形成一个像. 这些求和项都是正向延拓震源波场与反向延拓接收 点波场相互作用的结果. (7)式的第二个求和项中, 每 项都表示虚震源能量, 是经 $2 n$ 次反射的下行波能量, 且反向延拓反射 $2 n+3$ 次以上的能量, 两者进行互相 关. 这种互相关会产生无用的交叉串扰能量, 就像用 实际激发源进行标准偏移时, 源能量与多次波 (至少 反射了三次)互相关也会产生交叉串扰能量(图 2). (7) 式中, 所有物理上可能的波场相互作用都处在前两 
个求和项中. 从因果律角度考虑, 第三项中的互相关 不符合物理传播规律. 由源处正向传播得到的一阶 多次波永远不可能与由接收点处反向传播得到的一 阶多次波相遇. 通常来说, $n$ 阶多次波沿时间正延拓 后与 $m$ 阶或更低阶的多次波经反向延拓后, 进行互 相关不能成像, 因为这些同相轴在波场传播过程中 永远不可能相遇.

\section{4 地震多次波偏移与地震反射波偏移 2 范 数最小自适应匹配}

预测的多次波与原地震记录的多次波都含有高 阶多次波，这些不同阶的多次波在正向传播与反向 传播过程, 产生交叉串扰假象 (crosstalk). 为了压制 这种成像假象可以在数据域和成像域应用最小平方 算法, 因此, 本文在成像域应有了最小平方匹配技术. 最小平方的矩阵表达式为

$$
R(A)=\frac{1}{2}\left\|A M_{\text {multiple }}-M_{\text {reflect }}\right\|_{2},
$$

式中, $R(A)$ 为匹配残差, $A$ 为匹配系数, $M_{\text {multiple }}$ 为多次 波偏移成像结果, $M_{\text {reflect }}$ 为反射波偏移成像结果.

对(8)式求解可有多种办法, 这里采用最小平方 共轭梯度法求解, 共轭梯度法希望通过避免搜索过 的方向来寻找新的方向以提高计算效率, 该方法希 望在搜索中对每一个搜索方向只使用一次. 该方法 的关键是构建一组共轭的方向来替代直接使用梯度 方向.

$$
P^{k+1}=-g^{k+1}+\beta_{k} P^{k},
$$

$$
\beta_{k}=\frac{\left(P^{k}\right)^{T} Q g^{k+1}}{\left(P^{k}\right)^{T} Q p^{k}},
$$

式中, $P$ 表示共轭梯度方向, 其中 $g$ 表示其梯度方向, $\beta_{k}$ 表示加权系数, $k$ 是迭代次数. 应用迭代方法, 将多 次波与一次波能量匹配, 一次波无法照明的构造由 多次波补充.

\section{5 数值实验}

图 3 所示是 Sigsbee2B(Paffenholz 等, 2002)盐丘 模型. 模型有一系列沉积序列，同时发育着许多正断 层和逆冲断层. 该区域速度变化范围为 1437 4511 $\mathrm{m} \mathrm{s}^{-1}$. 水底、水面和盐体底部界面速度对比明显，可 以产生自由界面多次波和层间多次波(图 3). 由于盐 体的几何形态产生了多路径的非双曲时差, 导致了 传统一次反射波偏移照明度低的问题. 速度模型共 有 3201 (水平方向) $\times 1201$ (在深度方向)个网格点, 网 格间距在两个方向上均为 $7.62 \mathrm{~m}$. 总共有 496 个炮集, 炮间距为 $7.62 \mathrm{~m}$, 每个炮集有 348 道, 道间距为 7.62 $\mathrm{m}$, 采样总时间为 $12 \mathrm{~s}$, 采样间隔为 $8 \mathrm{~ms}$, 震源所在 深度与接收点相同, 为 $7.62 \mathrm{~m}$.

本文对 Sigsbee2B 模型数据进行测试, 对包含一 次波和多次波的地震数据进行逆时偏移. 传统逆时 偏移得到盐下成像结果如图 4 所示. 成像结果中存在 着严重的表面多次波和层间多次波产生的成像假象. 在复杂的覆盖层下照明度弱, 反转悬垂盐体下区域 和椭圆指示的区域未能成像, 倾斜断层也无法清晰
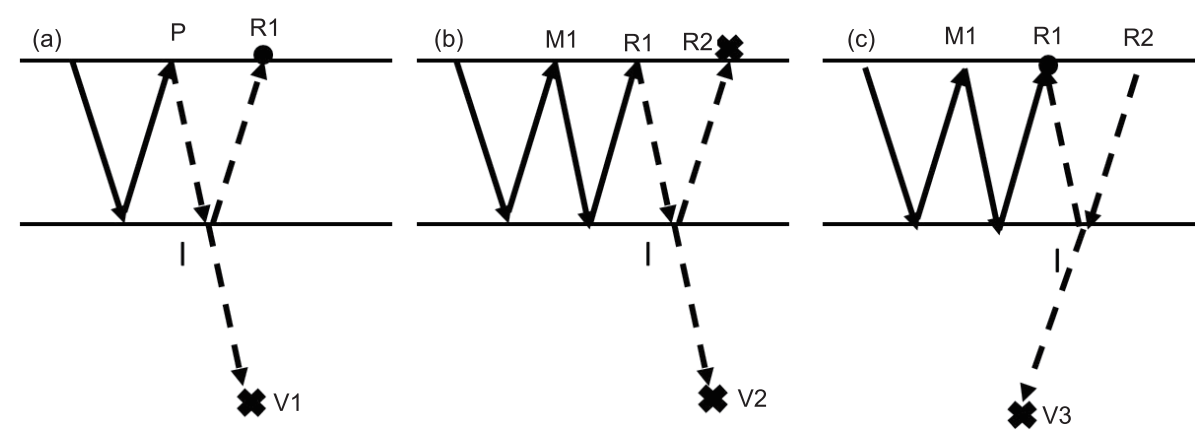

图 2 多次波成像假象示意图

(a) 多次波由地表 R1 点反向延拓到 I 点，与 $\mathrm{P}$ 点正向传播的反射波在 I 点相遇成像. 正向传播的反射波向下传播经过界面形成透射波到 V1 点. (b) R1 处的 1 阶多次波穿过界面 I 到达 V2. (c) R2 接收点的 2 阶多次波反向传播, 经过界面透射到 V3. V1, V2, V3 由于是透射波相遇形成假像 


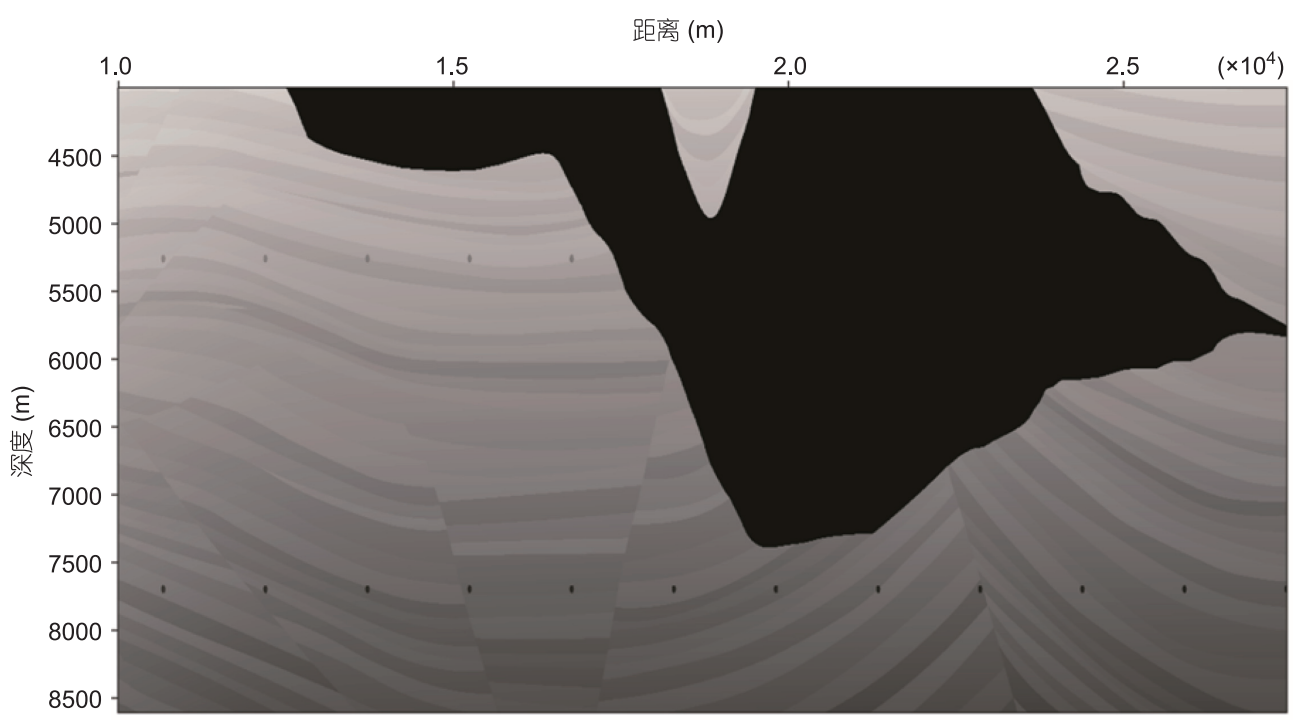

图 3 Sigsbee2B 二维盐丘模型

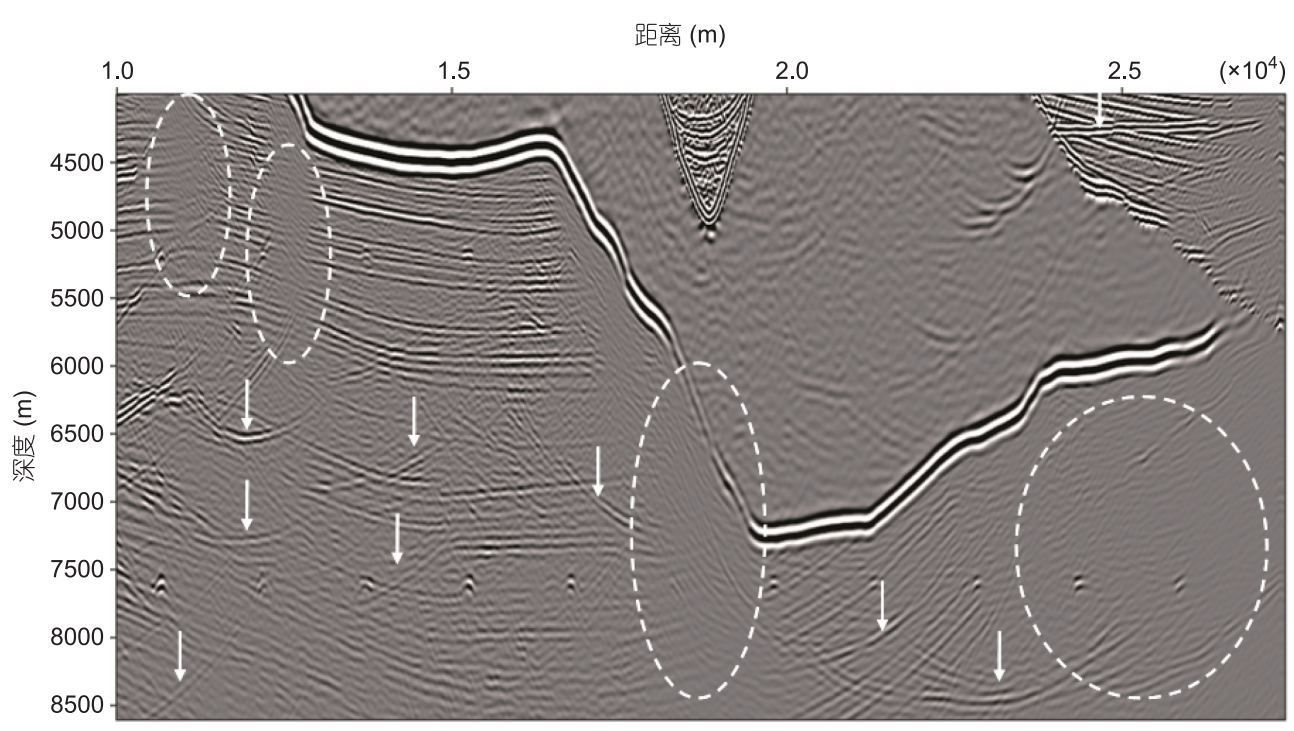

图 4 含有多次波的传统反射波逆时偏移

成像. 图 5 是传统反射波成像结果, 偏移之前已将多 次波压制，逆时偏移处理的是没有表层多次波的 Sigsbee2B 数据. 从而不会再有因表层多次波而产生 的假象(图 5). 图 5 表明只用反射波可以清晰对垂直 断层和悬垂界面成像, 但是椭圆所指定的悬垂盐体 以下区域则仍然由于低照明度而很难成像. 如果采 集系统不能提供适当的照明度, 即使有了再准确的 速度模型, 也不可能完整地对目的层成像. 因此, 为 提高地震波的照明度, 近些年来宽方位拖缆采集被 广泛应用, 而宽方位采集是非常昂贵又消耗时间的

\section{采集方式.}

为了得到更好的盐下成像结果，利用多次波对 Sigsbee2B 数据进行偏移(图 6), 表层多次波通过(1) 式方法预测得到. 多次波偏移能得到更清楚的断层 和界面轮廓, 界面和断层成像连续好, 尤其是模型右 下角区域，通过一次反射波是无法得到的. 多次波包 含的反射角度范围比一次波小, 通过图 6 可以看到, 多次波可用来提供更多的地下构造信息. 这就是因 为多次波偏移时有更加多样的射线路径. 图 4 中的很 多虚假同相轴是因多次波噪声产生. 但另一方面, 将 


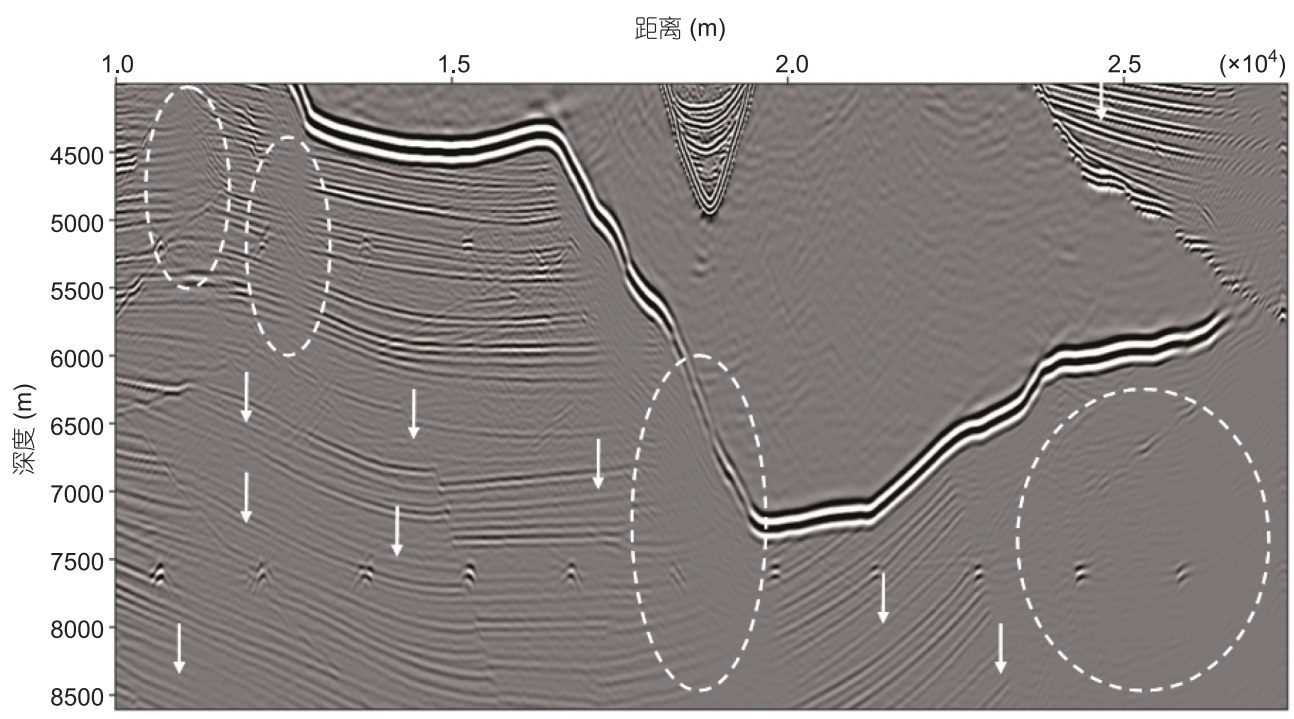

图 5 不含多次波的传统反射波逆时偏移

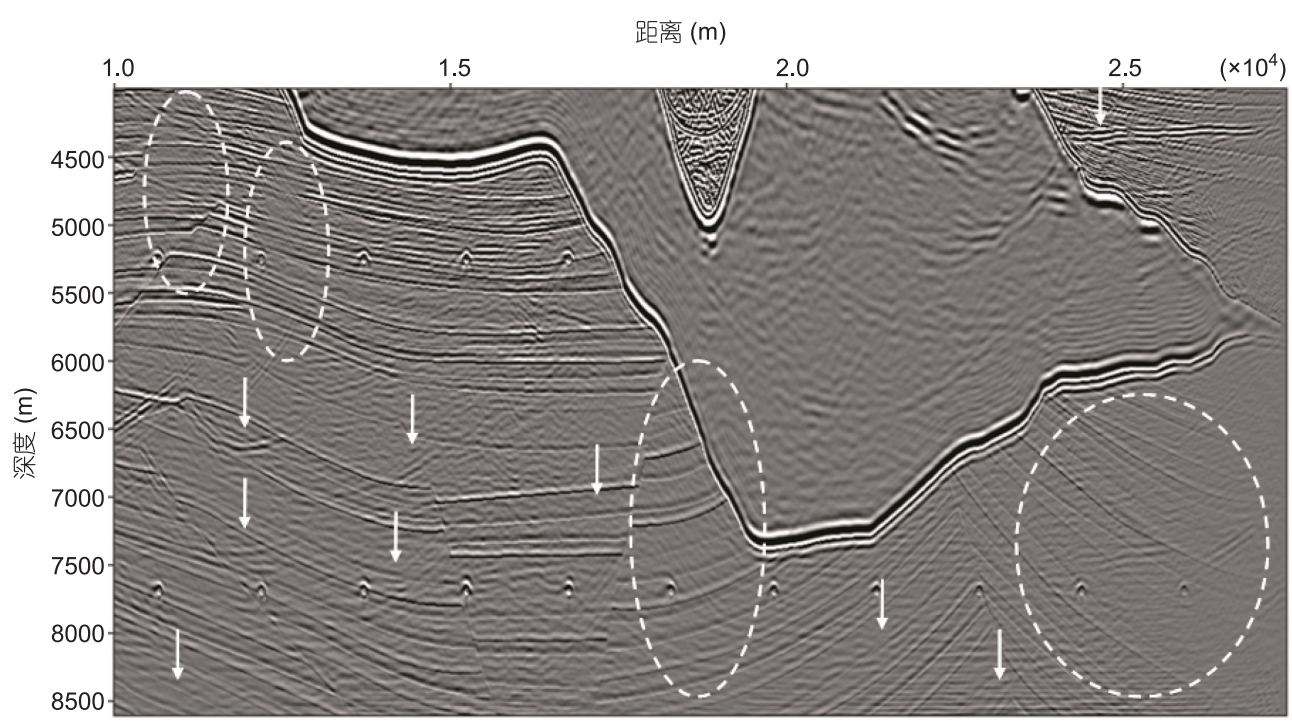

图 6 多次波逆时偏移

它应用到偏移处理中时, 却能够提够有价值的信息 从而改善盐下成像.

\section{6 南海深水多次波偏移}

将提出的多次波偏移方法应用到南海深水成像 研究. 所选测线是南海某区域二维测线. 测线长度 $48 \mathrm{~km}$, 总炮数为 1498 炮, 总道数为 539280 道, 每炮 360 道, 记录长度为 $8 \mathrm{~s}$, 采样间隔为 $2 \mathrm{~ms}$, 样点数为 4000. 图 7(a)是其中一炮地震记录, 图 7(b)是预测多
次波的单炮记录, 采集参数与原始道集相同.

首先应用与表面相关的多次波 SRME 方法预 测多次波，多次波的预测需要经过三个主要的步骤: 地震数据规则化、地震多次波的预测、预测多次波 与地震记录的匹配. 这三个步骤每一步都很重要, 缺一不可. 南海深水的数据不是规则化的数据, 需要 进行规则化处理. 通过地震道内插可以使得输入数 据的炮点位置和检波点位置重合. 对近偏移距的 缺失, 而实际数据缺失的近偏移距道需要通过记录 道外推来获得, 应用了基于 F-K 偏移和反偏移的地震 


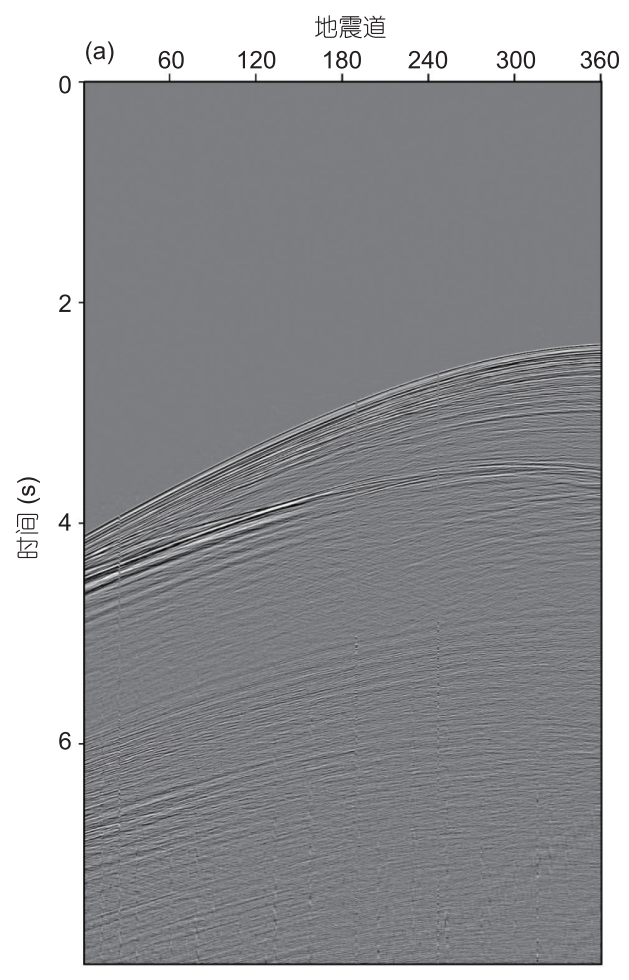

(b) 地震道

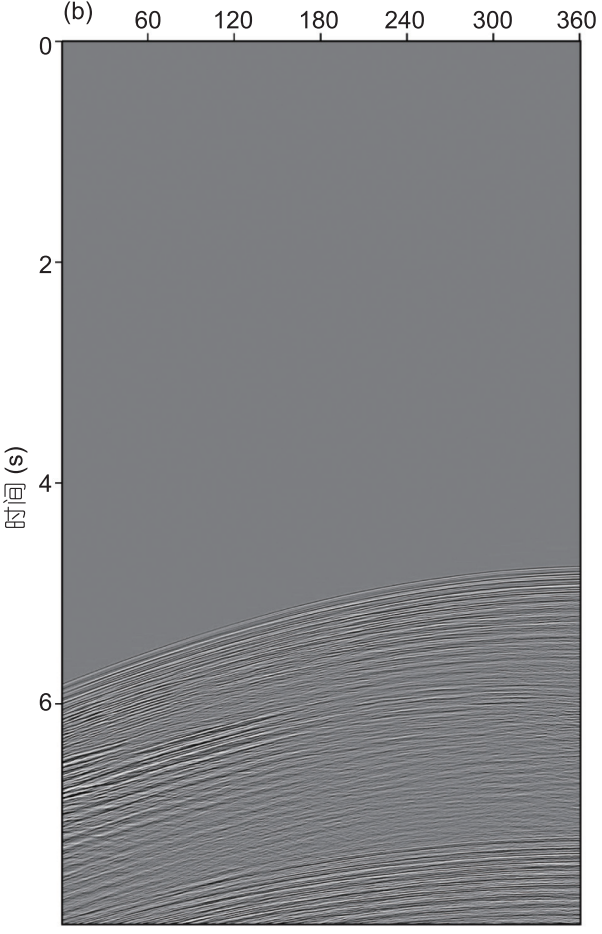

图 7 南海深水单炮地震记录(a)和南海深水预测多次波记录(b)

道插值法, 该方法是通过 F-K 偏移和反偏移的串联 使用来实现的. 预测部分, 在南海深水应用了反馈 迭代方法, 反馈迭代方法是一种完全数据驱动的 方法, 通过迭前直接反演来预测多次波, 其基本依据 是原始数据中包含了一次波和所有与反射界面有关 的多次波信息, 把这些波场作为新的源, 并利用记录 数据中所得到关于地下介质的信息, 则可以模拟 出多次波. 由于可供利用的信息不完整, 所以预测多 次波和实际记录中的多次波在到时、振幅和相位上存 在一些差异, 这种差异可以通过自适应匹配滤波来 消除。

图 8(b)与(a)结果类似, 通过匹配多次波的结果 加到反射波中, 弥补反射波的不足. 反射波剖面与多 次波与反射波匹配的剖面断层成像质量较好, 断面 清楚易于识别; 凹陷内部地震相反射结构及地层接 触关系更为清晰. 从剖面中可以看出, 凹陷缓坡带主 要发育多个次级反向断层, 易在缓坡背景下发育隆 上次洼结构, 从而控制着该部位地层沉积, 利于该地 区聚煤作用优势相带的发育, 从而可形成煤系烃源 岩; 整体上, 不同地层之间地震相包络特征差异明
显, 地震相由弱振幅、低频差连续性、过渡为中强振 幅、中低频、连续性一般, 进一步过渡为中弱振幅、 中高频、高连续性特征, 表现出构造活动性及沉积环 境之间的差异特征. 多次波偏移结果, 反射波结果及 其多次波与反射波匹配的偏移结果有一定差异(图 8(b)), 在浅层白色框中, 分辨率比图 8(a)和(b)高, 其 他区域连续性和聚焦性不如反射波和匹配多次波. 这是由于多次波偏移需要预测多次波, 而多次波预 测的准确度直接影响了成像质量. 当多次波结果与 反射波结果匹配时, 相同的同相轴会得到加强, 即结 果中反映地下真实存在的地层的位置会得到加强, 分 辨率和同相轴的连续性也会得到提高. 南海深水的速 度模型是由地震层析方法计算得到，该方法只能提供 一个低频光滑的速度模型, 给不出界面的位置, 对多 次波偏移也会产生影响. 图 9 是南海深水偏移的局部 放大结果，从放大图像可以看到反射波偏移(图 9(a)) 和多次波与反射波匹配(图 9(c))在 1.6 2.4 $\mathrm{km}$ 深度波 组清晰连续, 有较高的分辨率. 在 $2.6 \sim 4.0 \mathrm{~km}$ 深度偏 移结果有较好的连续性, 波型有较大的改善, 分辨率 有一定的提高. 

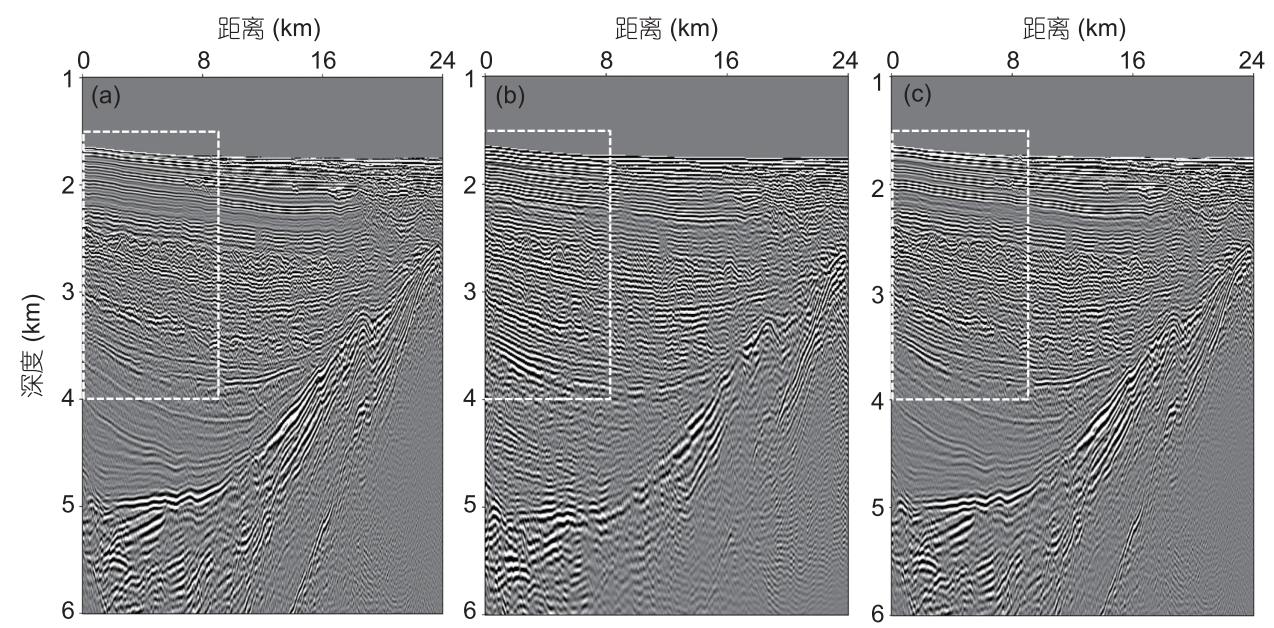

图 8 南海深水偏移结果图

（a) 南海深水反射波偏移结果; (b) 南海深水多次波偏移结果; (c) 南海深水反射波与多次波匹配偏移结果
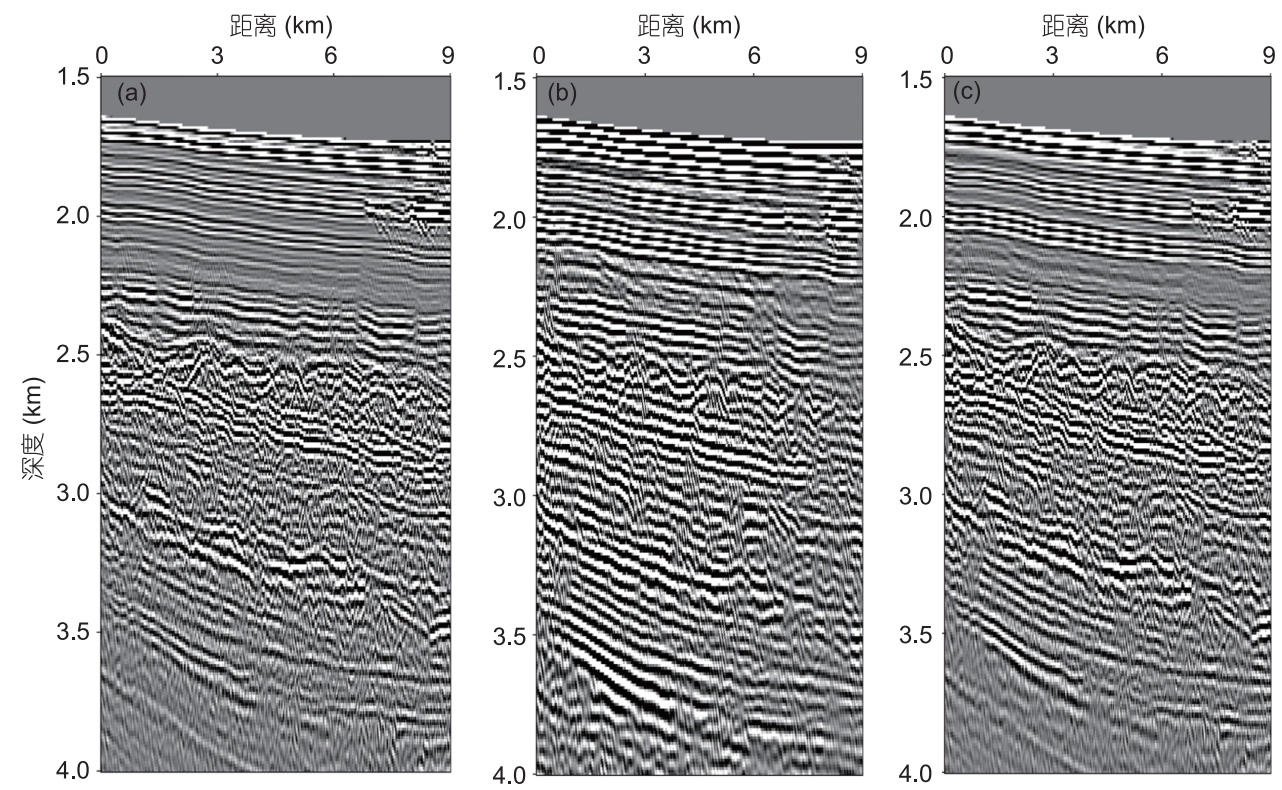

图 9 南海深水偏移的局部放大结果

(a) 南海深水反射波偏移局部放大; (b) 南海深水多次波偏移局部放大; (c) 南海深水反射波与多次波匹配偏移结果

\section{7 讨论与结论}

本文提出了多次波逆时偏移, Sigsbee2B 测试已 表明, 偏移多次反射波得到正确的界面位置是可能 的. Sigsbee2B 结果令人振奋, 但多次波逆时偏移仍 有一些问题需要解决. 大部分多次波偏移之后都提 供了有价值的盐下区域的信息从而弥补了低照明度 现象, 而且通过一次波很难做到这一点. 但要注意,
更高阶的多次波可能会与其他更高阶的多次波互相 关, 从而产生交叉串扰偏移假象. 出现这些虚假同相 轴, 是因为成像过程中做了不需要的多次波间的互 相关, 在速度对比明显时更为严重. 因用了传统成像 条件偏移, 更高阶多次波会导致形成假像. 要压制假 像, 需要降低各个阶多次波的无反射条件并且扩大 入射角. 多次波偏移技术可成为一个强大的工具, 提 供更大范围的、更多覆盖次数的、均衡的盐下照明度. 
本文提出的多次波偏移方法同常规偏移方向相比, 在一次波照明度很高, 常规一次波偏移能够得到 很好的结果时, 优势不明显, 但若由于观测系统的 布设以及地下构造复杂等原因, 一次波照明有限, 本文的方法便能体现出较明显的成像优势. 但多次 偏移带来的交叉串扰假象, 对速度模型的要求更高 等问题也需要进一步研究. 同时, 实际资料的测试局 限于为二维资料, 在三维情况下多次波的预测应该 更为准确, 下一步的研究工作也会扩展到三维资料 处理.
南海深水多次波偏移结果与一次反射波相近, 没有显示出多次波偏移的特别明显的优势. 主要原 因是实际资料的模型是由层析方法建立的宏速度模 型，宏速度模型是平滑模型，无法产生多次波界面. 在下一步的工作中，应该在宏速度模型基础上，应用 全波形反演, 获得更精确的速度模型, 这样多次波偏 移效果会大幅提高. 对于高速的盐下或者火山岩下 部地区，由于地震反射波不宜穿过，但多次波会在盐 下形成层间反射，照明高速体下部区域，更适合多次 波成像.

\section{参考文献}

Baysal E, Kosloff D D, Sherwood J W C. 1983. Reverse time migration. Geophysics, 48: 1514-1524

Berkhout A J, Verschuur D J. 1994. Multiple technology: Part 2, migration of multiple reflections. In: 64th, Annual International Meeting, SEG. 64: $1497-500$

Berkhout A J, Verschuur D J. 2003. Transformation of multiples into primary reflections. In: 2003 SEG Annual Meeting, 26-31 October, Dallas, Texas. 73: 1925-1928

Brown M P, Guitton A. 2005. Least-squares joint imaging of multiples and primaries. Geophysics, 70: s79-s89

Chang W, Mcmechan G A. 1987. Elastic reverse-time migration. Geophysics, 52: 1365-1375

Clearbout J F. 1968. Synthesis of a layered medium form its acoustic transmission response. Geophysics, 33: 264-269

Guitton A. 2002. Shot-profile migration of multiple reflections. In: Annual International Meeting, SEG. Expanded Abstracts, 72: 1296-1299

He R, Schuster G. 2003. Least-squares migration of both primaries and multiples. In: Annual International Meeting, SEG. 73: 1035-1038

Jiang Z Y, Sheng J M, Yu J H, et al. 2007. Migration methods for imaging different-order multiples. Geophys Prospect, 55: 1-19

Liu Y K, Chang X, Jin D G, et al. 2011. Reverse time migration of multiples for subsalt imaging. Geophysics, 76: 209-216

Muijs R, Robertsson J O A, Holliger K. 2007. Prestack depth migration of primary and surface-related multiple reflections: Part II-Identification and removal of residual multiples. Geophysics, 72: S71-S6

Paffenholz J, Mclain B, Zaske J, et al. 2002. Subsalt multiple attenuation and imaging: Observations from the Sigsbee2B synthetic dataset. In: Annual International meeting, SEG. 72: 2122-2125

Reiter E C, Toks M N, Keho T H, et al. 1991. Imaging with deep-water multiples. Geophysics, 56: 1081-1086

Schuster G T, Yu J, Sheng J, et al. 2004. Interferometric/daylight seismic imaging. Geophys J Int, 157: 838-852

Shan G. 2003. Source-receiver migration of multiple reflections. In: Annual International Meeting, SEG. 73: 1008-1011

Sheng J. 2001. Migration of multiples and primaries in CDP data by crosscorrelation migration. In: 71st Annual International Meeting, SEG. 71: 1297-1230

Vasconcelos I, Snieder R, Hornby B. 2008. Imaging internal multiples from subsalt VSP data-Examples of target-oriented interferometry. Geophysics, 73: S157-S168

Verschuurd D J, Berkhout A J, Wapenaar C P A. 1992. Adaptive surface-related multiple elimination. Geophysics, 57: 1166-1177

Verschuurd D J, Berkhout A J. 2005. Transforming multiples into primaries: Experience with field data. In: Annual International Meeting, SEG. 75: 2103-2106

Yu J, Schuster G. 2002. Joint migration of primary and multiple reflections in RVSP data. In: Annual International Meeting, SEG. 72: 2373-2376 